\title{
Melting and Crystallization of Ice in Partially Filled Nanopores
}

\author{
Estefanía González Solveyra, ${ }^{\dagger}$ Ezequiel de la Llave, ${ }^{\dagger}$ Damián A. Scherlis, ${ }^{\dagger}$ and Valeria Molinero*, \\ ${ }^{+}$Departamento de Química Inorgánica, Analítica y Química Física, Facultad de Ciencias Exactas y Naturales, \\ Universidad de Buenos Aires, Ciudad Universitaria, Buenos Aires, Pab II, C1428EHA, Argentina \\ ${ }^{\ddagger}$ Department of Chemistry, University of Utah, 315 South 1400 East, Salt Lake City, Utah 84112-0850, United States
}

\begin{abstract}
We investigate the melting and formation of ice in partially filled hydrophilic and hydrophobic nanopores of $3 \mathrm{~nm}$ diameter using molecular dynamics simulations with the $\mathrm{mW}$ water model. Above the melting temperature, the partially filled nanopores contain two water phases in coexistence: a condensed liquid plug and a surface-adsorbed phase. It has been long debated in the literature whether the surface-adsorbed phase is involved in the crystallization. We find that only the liquid plug crystallizes on cooling, producing ice I with stacks of hexagonal and cubic layers. The confined ice is wetted by a premelted liquid layer that persists in equilibrium with ice down to temperatures well below its melting point. The liquid-ice transition is first-order-like but rounded. We determine the temperature and enthalpy of melting as a function of the filling

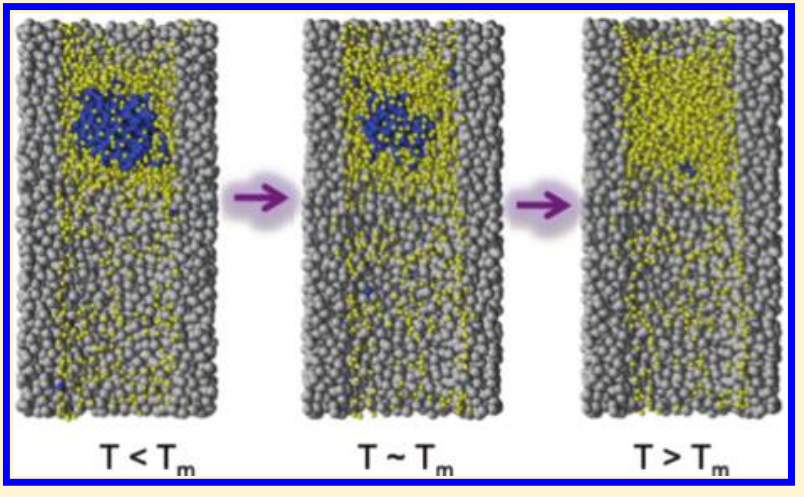
fraction of the pore. In agreement with experiments, we find that the melting temperature of the nanoconfined ice is strongly depressed with respect to the bulk $T_{\mathrm{m}}$, it depends weakly on the filling fraction and is insensitive to the hydrophobicity of the pore wall. The state of water in the crystallized hydrophilic and hydrophobic pores, however, is not the same: the hydrophobic pore has a negligible density of the surface-adsorbed phase and higher fraction of water in the ice phase than the hydrophilic pore. The widths of the ice cores are nevertheless comparable for the hydrophobic and hydrophilic pores, and this may explain their almost identical melting temperatures. The enthalpy of melting $\Delta H_{\mathrm{m}}$, when normalized by the actual amount of ice in the pore, is indistinguishable for the hydrophobic and hydrophilic pores, insensitive to the filling fraction, and within the error bars, the same as the difference in enthalpy between bulk liquid and bulk ice evaluated at the temperature of melting of ice in the nanopores.
\end{abstract}

\section{INTRODUCTION}

The phase behavior of water in confined geometries has attracted significant interest due to the ubiquity of confined water in nature and materials, and the fundamental differences between ice-liquid equilibrium for water in bulk and in confinement. ${ }^{1,2}$ Mesoporous silica materials MCM-41 and SBA- 15 provide arrays of monodisperse cylindrical pores with tunable diameter between 2 and $10 \mathrm{~nm}$. These materials can be functionalized to tune the hydrophobicity of the pore wall and are widely used in studies of confined water. The freezing and melting of water in filled and partially filled pores of MCM-41 and SBA-15 silica have been extensively characterized through differential scanning calorimetry (DSC), neutron (ND) and X-ray diffraction, NMR, and dielectric measurements. ${ }^{3-14}$ The most important results that emerge from these studies are a significant depression of the melting temperature with respect to the value for bulk ice and the existence of noncrystallizable water in the pores, even at temperatures well below the melting point of the confined ice.

Differential scanning calorimetry demonstrates that the melting temperature of confined ice in pores with diameter $3 \mathrm{~nm}$ or larger is rather insensitive to the hydrophilicity/hydrophobicity of the pore wall and the degree of filling of the pore. ${ }^{2,3,11,15,16}$ Interestingly, a recent X-ray and neutron diffraction study of water in partially filled hydrophilic and hydrophobic nanopores at $298 \mathrm{~K}$ showed significant differences in the structure of water in the two environments, ${ }^{17}$ although the melting temperatures of ice in the hydrophilic and hydrophobic nanopores were essentially identical. ${ }^{11}$ This poses the question of whether the state of water at temperatures below the melting point is the same in hydrophobic and hydrophilic nanopores.

The melting temperature of ice in cylindrical nanopores is well represented by a modified Gibbs-Thompson (GT) equation, $\Delta T_{\mathrm{m}}=K_{\mathrm{GT}} /(R-d)$, which predicts an inverse relation between the depression of the melting temperature with respect to the bulk value, $\Delta T_{\mathrm{m}}$, and the effective radius $R-d$ of the ice confined in a pore of radius $R^{2,18,19}$ This expression suggests the presence of a noncrystallized water layer of width $d$ in coexistence with the confined ice just below $T_{\mathrm{m}} \cdot{ }^{2-5}$ DSC and NMR studies of water in partially filled ordered silica pores MCM-41 and SBA-15 with radius between 2 and $10 \mathrm{~nm}$ yield melting temperatures satisfactorily fitted by the GT equation with $K_{\mathrm{GT}}=52.4 \pm 0.6 \mathrm{~K} \mathrm{~nm}$ and $d=0.6 \pm 0.1 \mathrm{~nm}^{3} K_{\mathrm{GT}}$ determined from the experiments is in excellent agreement with the one predicted from bulk water

Special Issue: H. Eugene Stanley Festschrift

Received: $\quad$ May 29, 2011

Revised: July 16, 2011

Published: August 24, 2011 
properties at $273 \mathrm{~K}$ under the assumption of complete wetting of the pore wall by the liquid layer, ${ }^{18,19} K_{\mathrm{GT}}=2 T_{\mathrm{m}} \gamma v / \Delta H_{\mathrm{m}}=51.9 \pm$ $4 \mathrm{~K} \mathrm{~nm}$, where $\Delta H_{\mathrm{m}}$ is the enthalpy of melting, $\mathrm{v}$ the molar volume, $\gamma$ the liquid-ice surface tension for bulk water. ${ }^{3}$ There is consensus that this agreement probably results from compensation between decreased values of the enthalpy of melting $\Delta H_{\mathrm{m}}$ and liquid-ice surface tension $\gamma$ in the nanopore. ${ }^{2,3}$ There is no consensus, however, on the value of the enthalpy of melting per mol of confined ice, ${ }^{3,6,15}$ because of the uncertainty in assigning what fraction of the water belongs to the ice phase just below the melting temperature.

NMR, neutron diffraction, and X-ray diffraction experiments evidence the presence of a disorganized state of water in silica nanopores at temperatures below the melting points of ice in the pores. ${ }^{11-14,16}$ Diffraction patterns of water in mesoporous silica indicate that this disorganized water component is in reversible equilibrium with the confined ice. ${ }^{11,13,14}$ On the basis of NMR relaxation times and neutron diffraction measurements for water and ice confined in SBA-15 silica, Webber et al. proposed that this disordered component is ice in a plastic state, with substantial rotational motion and a well-defined crystal structure. ${ }^{13}$ The calorimetric results, on the other hand, suggest that there is a layer of liquid between the ice and the pore wall. State of the art experimental methods, however, are not yet able to resolve the structure and spatial distribution of the different components of water in the pore.

Molecular simulations provide optimum spatial resolution for the study of the structure of water within nanopores and the phase transformations it experiences on cooling and heating. Molecular simulations have predicted novel phases of ice in carbon nanotubes ${ }^{20-22}$ and revealed a variety of ice forms for water confined between parallel surfaces. ${ }^{23-38}$ The crystal structures of ice confined in narrow carbon nanotubes and between parallel plates are, for the most part, distinct from any of the bulk forms of ice, whereas neutron and X-ray diffraction experiments indicate that the ice formed in silica and functionalized silica nanopores is a hybrid of ice I with features of the cubic and hexagonal polymorphs. ${ }^{16,39-41}$ There has been a study of the melting of nanocolumns of hexagonal ice in vacuum ${ }^{42}$ but, to the best of our knowledge, no study of melting or freezing of ice in cylindrical silica or silica-like nanopores using atomistic simulations. This scarcity is mostly due to the high computational cost of the long simulations required for the rare ice nucleation event and equilibration of systems that contain at least several thousand molecules. Recently, we presented the first study of the freezing, melting, and structure of ice in a fully filled $3 \mathrm{~nm}$ hydrophilic nanopore using molecular dynamics simulation with a very efficient coarse-grained model of water, $\mathrm{mW} .{ }^{43}$ The $\mathrm{mW}$ model represents each water molecule as a single particle with short-ranged anisotropic interactions that mimic hydrogen bonds and is $\sim 180$ times more efficient than fully atomistic models of water with Ewald sums. ${ }^{44}$ The $\mathrm{mW}$ model reproduces the anomalies and structure of liquid water and its phase transformations, including the melting temperatures of hexagonal and cubic ice I, and the kinetics of crystallization of bulk ice. ${ }^{4-46}$ Our study of water in the nanopore provided direct evidence for the existence of a premelted liquid layer between the pore-wall and the confined ice in the pores, showed that nanoconfined ice contains an almost random arrangement of cubic and hexagonal layers, in a ratio approximately 2 to 1 , and revealed that the crystallization was initiated by ice nuclei without well-defined stacking structure and containing about 100 water molecules. ${ }^{43}$
The melting temperature for the confined ice predicted by the simulations, $T_{\mathrm{m}}=222 \pm 3 \mathrm{~K},{ }^{43}$ was in very good agreement with the one measured or predicted by the Gibbs-Thomson equation with the experimentally determined parameters, $215<$ $T_{\mathrm{m}}<231 \mathrm{~K}^{3-5}$

In the present work, we use coarse-grained molecular dynamics simulations to investigate the freezing, melting, and structure of water in $3 \mathrm{~nm}$ diameter partially filled cylindrical nanopores with hydrophobic and hydrophilic walls. The use of partially filled pores is crucial to investigate the effect of reduced dimensions of the liquid domain on the freezing and melting of water and to address whether the surface-adsorbed water phase in equilibrium with the liquid plays any role in the crystallization of water, an open question in the literature. ${ }^{5,16}$ The use of hydrophilic (silica-like) and hydrophobic walls allows us to address whether there is a correlation between the water-wall interaction, the structure of water in the pore and the temperature and enthalpy of melting of the confined ice.

\section{MODELS AND METHODS}

Systems. We analyze the crystallization and melting of water in partially filled hydrophilic and hydrophobic pores of $3 \mathrm{~nm}$ diameter. The fraction of water in the hydrophilic pore was $f=0.4,0.6,0.9$, and 1 , where the last corresponds to a pore fully filled with water. The hydrophobic pore was studied only at 0.6 filling fraction. The particles making up the hydrophilic pore wall and those contained within it were both modeled with the $\mathrm{mW}$ water force field, as in previous studies of melting ${ }^{43}$ and capillary condensation. ${ }^{47}$ Partially filled cylindrical nanopores were built according to the protocol of ref 47: first, a block of liquid water with dimensions $5 \mathrm{~nm} \times 5 \mathrm{~nm} \times 10 \mathrm{~nm}$ was equilibrated at $298 \mathrm{~K}$ and $1 \mathrm{~atm}$ (density $0.997 \mathrm{~g} \mathrm{~cm}^{3}$ ). A configuration of the liquid was randomly selected, wherein we defined a cylinder of radius $R_{\mathrm{p}}=1.5 \mathrm{~nm}$ aligned with the $z$-axis. The water molecules outside the cylinder were considered to be the pore wall and they were restrained to their original configuration through soft harmonic bonds with constant $k=30 \mathrm{kcal} / \AA^{2}$ between pore particles, in addition to water-water nonbonding interactions. ${ }^{47}$ This results in $3 \mathrm{~nm}$ diameter and $10 \mathrm{~nm}$ long pores lined with a surface that has the structure of liquid water at $298 \mathrm{~K}$ (the intermolecular interactions between the particles in the pore wall and the water molecules inside are described in the following subsection). By this procedure, $N_{\mathrm{T}}$ water molecules remained inside the cylinder. To produce varied pore filling fractions $f$, we removed $(1-f) N_{\mathrm{T}}$ water molecules from the water cylinder. The fully filled pore, $f=1$ was the same used in the study of ref 43 . A difference between the fully filled and partially filled pores, is the $\mathrm{T}$-shape of the liquid phase in the former, with a $\sim 1 \mathrm{~nm}$ head block of water phase to allow for the expansion and compression of the liquid upon cooling. The partially filled pores do not need of a slab space for expansion of the water. Further details regarding the building of the nanopores can be found in refs 43 and 47 . The number of water molecules inside each pore is indicated in Table 1.

Force Fields. Water was modeled with the monatomic water model $\mathrm{mW}^{44,47}$ that has been successfully employed in the study of liquid-crystal equilibrium in bulk and in nanopores. ${ }^{43,45,46}$ Each water molecule is modeled as a single particle with intermolecular interactions described by a Stillinger-Weber potential ${ }^{48}$ 
Table 1. Temperature and Enthalpy of Melting of Ice in Partially Filled Pores

$\begin{array}{rcccccc}f & \text { water-wall interaction } & \text { water molecules melting } & \text { total water molecules } & T_{\mathrm{m}}[\mathrm{K}] & \Delta H_{\mathrm{m}}{ }^{\mathrm{w} b}[\mathrm{kcal} / \mathrm{mol} \text { water }] & \Delta H_{\mathrm{m}}{ }^{\text {ice } c}[\mathrm{kcal} / \mathrm{mol} \text { of ice }] \\ 0.9 & \text { hydrophilic } & 754 & 2123 & 219 \pm 2 & 0.33 \pm 0.05 & 0.92 \pm 0.14 \\ 0.6 & \text { hydrophilic } & 475 & 1399 & 216.5 \pm 2 & 0.32 \pm 0.05 & 0.95 \pm 0.14 \\ 0.6 & \text { hydrophobic } & 591 & 1378 & 216 \pm 2 & 0.41 \pm 0.06 & 0.95 \pm 0.14 \\ 0.4 & \text { hydrophilic } & 211 & 948 & 212 \pm 2 & 0.23 \pm 0.03 & 1.04 \pm 0.14 \\ \text { bulk }^{a} & & & & 274 \pm 1 & 1.26 \pm 0.03 & 1.26 \pm 0.01\end{array}$

${ }^{a}$ Data for the mW model from ref 44, experimental values are $\Delta H_{\mathrm{m}}=1.44 \mathrm{kcal} / \mathrm{mol}$ and $T_{\mathrm{m}}=273.15 \mathrm{~K}^{72}{ }^{b}$ The suffix $w$ corresponds to the enthalpy of melting calculated by considering all the water molecules in the system. ${ }^{c}$ The suffix ice refers to the value normalized with the water molecules in the ice phase (including cubic, hexagonal, and interfacial ice).

that consists of a sum of pairwise $\phi_{2}(r)$ and three-body $\phi_{3}(r, \theta)$ contributions,

$$
\begin{aligned}
& E=\sum_{i} \sum_{j>1} \phi_{2}\left(r_{i j}\right)+\sum_{i} \sum_{j \neq 1} \sum_{k>j} \phi_{3}\left(r_{i j}, r_{i k}, \theta_{i j k}\right) \\
& \phi_{2}\left(r_{i j}\right)=A \varepsilon\left[B\left(\frac{\sigma}{r_{i j}}\right)^{p}-\left(\frac{\sigma}{r_{i j}}\right)^{q}\right] \exp \left(\frac{\sigma}{r_{i j}-a \sigma}\right) \\
& \phi_{3}\left(r_{i j}, r_{i k}, \theta_{i j k}\right)=\lambda \varepsilon\left(\cos \theta_{i j k}-\cos \theta_{0}\right)^{2} \exp \left(\frac{\gamma \sigma}{r_{i j}-a \sigma}\right) \exp \left(\frac{\gamma \sigma}{r_{i k}-a \sigma}\right)
\end{aligned}
$$

where $A=7.049556277, B=0.6022245584, p=4, q=0, \gamma=1.2$, $a=1.8, \theta_{\mathrm{o}}=109.47^{\circ}$, the characteristic size $\sigma=2.3925 \AA$, the characteristic energy $\varepsilon=6.189 \mathrm{kcal} \mathrm{mol}^{-1}$, and the weight of the three-body interactions $\lambda=23$.15. The model encourages "hydrogen bonded" configurations through the nonbonding three-body term, which penalizes water-water-water angles departing from the ideal tetrahedral angle $\theta_{\mathrm{o}}=109.5^{\circ}$. The mass of a mW particle corresponds to that of a water molecule, $18.015 \mathrm{~g} / \mathrm{mol}^{4}{ }^{44}$

In spite of not having hydrogen atoms or electrostatic interactions, the $\mathrm{mW}$ model reproduces the experimental enthalpy of vaporization and density of the liquid, radial and angular distribution functions of the liquid, the liquid-vapor surface tension, the melting temperature of hexagonal ice (and correctly predicts that cubic ice is marginally less stable), and the excess free energy of liquid with respect to ice. ${ }^{44,49}$ The enthalpy and entropy of melting of ice with the $\mathrm{mW}$ model is underestimated by $13 \%$ with respect to the experimental value. The agreement between the values predicted by $\mathrm{mW}$ and the experiments for all these properties are comparable to or better than those for any of the most popular atomistic models of water (SPC, SPCE, TIP3P, TIP4P, TIP5P) ${ }^{44}$ and comparable to the one for TIP4P/ice. ${ }^{50}$ The time scales of molecular processes in the mW model, however, are shorter than for atomistic models because the model lacks the hydrogen atoms that effectively add a friction to the mobility of the center of mass of the water molecules. We refer the interested reader to ref 44 for further details on the mW model.

The hydrophobic pore wall is constructed in identical manner to the hydrophilic one, carving a block of liquid water at $298 \mathrm{~K}$ and adding soft restraining bonds between the pore wall particles to keep them in the positions they would have in the original liquid water configuration. The only difference between the hydrophilic and hydrophobic pore is on the parameters of the interaction potential (eq 1) between the water molecules inside the pore and the atoms in the wall: in the hydrophobic pore the wall makes no hydrogen bonds with water (i.e., the weight of the three body term in the potential is zero, $\lambda=0$, for triplets involving water and pore wall molecules) and it presents little attraction with water, $\varepsilon_{\mathrm{wp}}=0.2 \mathrm{kcal} \mathrm{mol}^{-1}$ (all other parameters for the interaction of water with the particles of the hydrophobic pore are as indicated above for water). These parameters correspond to the most hydrophobic surface that still prevents dewetting of the pore, as shown elsewhere. ${ }^{51}$ The waterwall interactions of the hydrophilic pore are described by eq 1 with all parameters identical to those used for water-water interactions. ${ }^{43,47}$

Simulation Methods. Molecular dynamics simulations of the fully filled and partially filled pores were carried out using LAMMPS. $^{52}$ The equations of motion were integrated in the canonical (NVT) ensemble using the velocity Verlet algorithm with a time step of $10 \mathrm{fs}$. The temperature was controlled with a Nose-Hoover thermostat with relaxation time 0.25 ps. The systems were simulated with periodic boundary conditions in the three dimensions. Simulation times vary between $50 \mathrm{~ns}$ and $2 \mu \mathrm{s}$ and are indicated through the text.

Identification of Ice. For the distinction of liquid from crystal and the identification and quantification of hexagonal and cubic ice during crystallization and melting, we use the CHILL algorithm, which allows for the identification of ice from liquid water and distinguishes the cubic and hexagonal ice polymorphs in a rotational invariant manner. ${ }^{43}$ The CHILL algorithm determines the correlation of orientations between the first coordination shells of neighboring water molecules to classify them according to whether they present the local order of cubic ice (C), hexagonal ice $(\mathrm{H})$, interfacial or intermediate ice (I), or liquid (L). Molecules classified as $\mathrm{C}$ or $\mathrm{H}$ have four tetrahedrally coordinated neighbors in their first coordination shell, each of them with four neighbors that have also themselves a tetrahedral first shell. The difference between $\mathrm{C}$ and $\mathrm{H}$ is that $\mathrm{C}$ has four staggered intermolecular bonds (i.e., the orientation between the tetrahedron around $\mathrm{C}$ and the tetrahedral around each of its four neighbors is in a staggered conformation) whereas $\mathrm{H}$ has three bonds in a staggered conformation and one bond in an eclipsed conformation. We refer to the sum of $\mathrm{C}$ and $\mathrm{H}$ as core ice. Water molecules classified as interfacial or intermediate ice (I) have a tetrahedral first coordination shell but not the requisites to be $\mathrm{C}$ or $\mathrm{H}$ : I molecules have either two staggered bonds and at least one neighbor with two staggered bonds or three staggered bonds, no eclipsed bond and at least one neighbor with two staggered bond. These I molecules are found at the surface of crystallites and also in very deeply supercooled water, where they form threads. ${ }^{43,46}$ We refer the interested reader to ref 43 for the description and validation of the algorithm. We denote as ice core the sum of cubic and hexagonal ice, and as total ice, the sum of cubic, hexagonal and interfacial ice. To characterize individual ice nuclei, the water molecules that belong to core ice $(\mathrm{C}$ and $\mathrm{H})$ are clustered using as cutoff distance $3.5 \AA$, the first minimum of the water-water radial distribution function. The VMD program was used for visual analysis of the data. ${ }^{53}$ 
Exchange Rates. The exchange rate between molecules in the liquid and crystal phases was characterized by the probability of survival of molecules in each phase and by analyzing the translational motion in the liquid phase. The probability that a molecule survives in the phase $x$ (ice or liquid) for a time $t$ was calculated as

$$
P_{x}(t)=\frac{1}{I_{\mathrm{T}}} \sum_{\tau=1}^{I_{\mathrm{T}}} \frac{N(\tau, \tau+t)}{M(\tau)}
$$

where $N(\tau, t+\tau)$ is the number of water molecules that remain in phase $x$ during the time interval between $\tau$ and $\tau+\mathrm{t}, M(\tau)$ is number of molecules in such phase at time $\tau$, and $I_{\mathrm{T}}$ is the number of time intervals averaged over. $P(t)$ was analyzed in terms of stretched exponential decay with time,

$$
P(t)=A \mathrm{e}^{-(t / \tau) \beta}
$$

The correlation time is defined by

$$
\bar{\tau}=(\tau / \beta) \Gamma(1 / \beta)
$$

where $\Gamma$ is the gamma function. The translational motion of liquid water along the axis of the pore was analyzed qualitatively, comparing the axial position of the molecules at the beginning and at the end of each simulation.

\section{RESULTS AND DISCUSSION}

A. Crystallization of Water in Partially Filled Pores. The initial step of this study involved the crystallization of the partially filled pores. The initial configurations contained two phases of water in the pore: a liquid plug and a surface-adsorbed phase. At $298 \mathrm{~K}$, the surface-adsorbed phase in the hydrophilic nanopores has a surface density $\Gamma$ of about 3 water molecules per $\mathrm{nm}^{2}$ and is formed by small clusters, most of them containing less than five water molecules. ${ }^{47}$ The surface density for water adsorbed in the hydrophilic nanopores equilibrated for $200 \mathrm{~ns}$ at $200 \mathrm{~K}$ is comparable to the surface density $\Gamma$ at $298 \mathrm{~K}$. We do not find a dependence of $\Gamma$ with the filling fraction $f$, implying that the length of the cylindrical liquid plugs does not significantly affect the chemical potential of water. The surface-density $\Gamma$ of the hydrophobic nanopore is too small to be quantified: all the water has condensed in the liquid plug.

The crystallization of each partially filled pore was performed in two steps: First, the pore containing liquid water was cooled at a rate of $0.2 \mathrm{~K} \mathrm{~ns}^{-1}$ from $230 \mathrm{~K}$ down to $180 \mathrm{~K}$. This process resulted in ice nucleation in all pores, except for the one with the lowest filling fraction, $f=0.4$, for which an additional $2 \mu \mathrm{s}$ simulation at $180 \mathrm{~K}$ was needed to nucleate and grow ice. In a second step, the ice formed at $180 \mathrm{~K}$ was further annealed for $50 \mathrm{~ns}$ at $190 \mathrm{~K}$ followed by $50 \mathrm{~ns}$ at $200 \mathrm{~K}$ to complete the growth and favor its structural reorganization into a single crystallite. The result in all cases was an ice plug that occluded the pore. Crystallization of the $f=0.6$ and 0.9 partially filled pores over the cooling ramps yielded ice cores containing about a quarter of the water in the pores. Crystallization of water in silica nanopores with as little as 0.2 filling fraction has been observed in experiments. ${ }^{11}$ Due to the stochastic nature of the nucleation process, crystallization of water occurs more readily (i.e., at higher temperatures on constant cooling rate) on pores with a larger filling fraction. A decrease in the freezing temperature on decreasing the filling fraction been previously reported in a DSC study for water confined in SBA-15. ${ }^{16}$

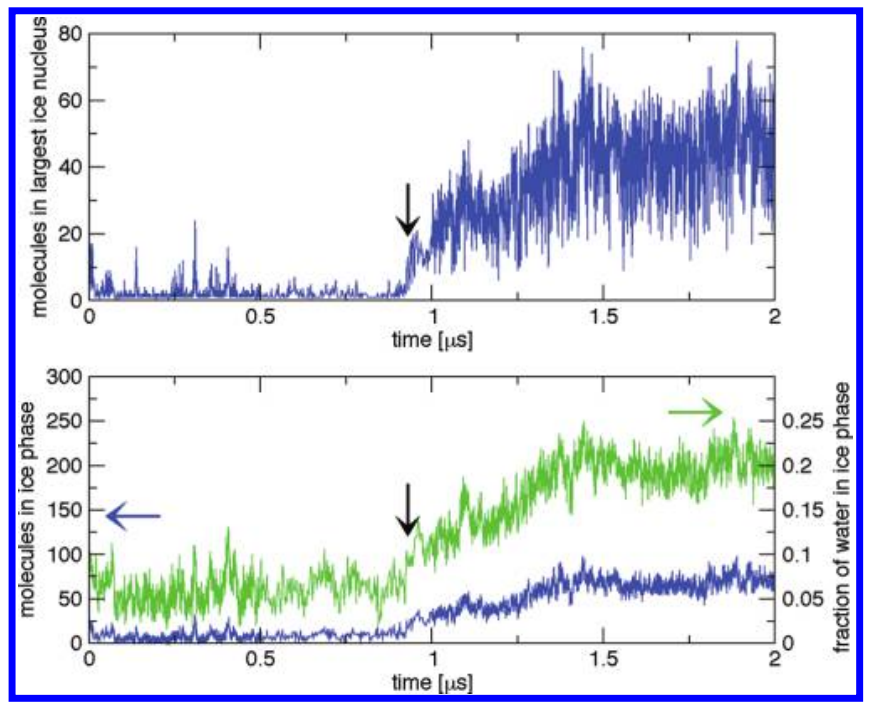

Figure 1. Crystallization of ice in a $40 \%$ filled hydrophilic pore at $180 \mathrm{~K}$. Upper panel: number of water molecules in the largest ice nucleus, counting only the molecules in the core (cubic and hexagonal ice) of the crystallite, as a function of time. The black arrow indicates the time at which the critical nucleus forms, ending the nucleation period and starting the period of growth of the crystallite. The maximum size of the subcritical nuclei suggests that the critical nucleus size contains a core of about 20 water molecules. Lower panel: The blue curve (label in left axis) shows the evolution of the number of water molecules in the core of the ice nuclei ( $\mathrm{C}$ and $\mathrm{H}$ ). The green curve (label in right axis) shows the total fraction of water in the ice phase, including cubic, hexagonal, and intermediate ice. Although there is about $5-10 \%$ intermediate ice in the nucleation period, intermediate ice does not form compact ice nuclei but loose threads ${ }^{46}$ that form and dissolve without leading to the growth of the crystallite until a sufficiently large "core" ice nucleus forms.

The crystallization of water occurs exclusively within the liquid plug. The surface-adsorbed molecules present in the hydrophilic pore do not form ice, consistent with their low surface density and the strong energy of adsorption to the walls $(6.83 \pm 0.08$ $\mathrm{kcal} / \mathrm{mol}$ at $298 \mathrm{~K}$ and $7.28 \pm 0.12 \mathrm{kcal} / \mathrm{mol}$ at $0 \mathrm{~K}$; see ref 47$)$. The crystallization was initiated by homogeneous nucleation (i.e., without assistance by the pore walls) from an ice nucleus that grew to yield a single crystallite, as previously reported for the nucleation of the filled pore in ref 43 . The size of the final ice crystallite strongly depended on the amount of water in the pore.

We analyze in detail the isothermal crystallization of the least filled pore, $f=0.4$, as it provides insight into the process of nucleation of ice from a very small pool of water molecules in a confined environment. Figure 1 shows the number and fraction of water molecules in the ice phase (upper panel) and the size of the largest ice nucleus (lower panel) for the pore with $f=0.4$ evolved at $180 \mathrm{~K}$. There are two well-defined regimes: an induction period during which subcritical ice nuclei, each containing less than $\sim 20$ water molecules in their core, form and dissolve until a critically sized nucleus is created, followed by a growth period in which the viable nucleus develops into a crystallite. The induction period for the nucleation is stochastic, taking about $900 \mathrm{~ns}$ in the simulation presented in Figure 1. This is 3 orders of magnitude longer than the $\sim 1-2$ ns induction period determined for the nucleation of ice in simulations of bulk $\mathrm{mW}$ water with 32768 molecules at the same temperature. ${ }^{46}$ The slower nucleation rate in the partially filled nanopore can be ascribed to two factors. First, the lowest driving force for ice 
nucleation in the pore, as the melting point of ice in the pore is depressed about $60 \mathrm{~K}$ with respect to the bulk value (see subsection $\mathrm{C}$, below). Second, nucleation is hindered by the scarcity of water in the liquid plug from which the ice nucleates in the partially filled pore. There are less than 1000 water molecules in the $f=0.4$ nanopore, about 650 of which are part of the liquid plug that nucleates the ice. As a comparison, the maximum density of ice nuclei in bulk water at $180 \mathrm{~K}$, attained when $\sim 40 \%$ of the water has crystallized, corresponds to approximately one ice nucleus every 800 water molecules. ${ }^{46}$ Ice nuclei containing as many as 20 molecules in their core are unable to trigger the crystallization in the $f=0.4$ pore at $180 \mathrm{~K}$ (upper panel of Figure 1). This suggests that at $180 \mathrm{~K}$ the critical nucleus contains about 20 molecules in its core, comparable to the $\sim 10$ estimated for the crystallization of bulk water at the same temperature. $^{46}$

The growth of ice in the partially filled pore with $f=0.4$ at $180 \mathrm{~K}$ spans over $500 \mathrm{~ns}$, about twice the growth time for ice in bulk water at the same temperature. ${ }^{46}$ The lengthening of the growth period is probably due to a slowdown of water mobility in confinement. The growth of ice in the pore at $180 \mathrm{~K}$ is about 25 times slower than at $195 \mathrm{~K}^{43}$ The crystal produced in the partially filled pore is fairly small, attaining a maximum of about 50 water molecules in its core (200 molecules when including the interfacial ice) over the $2 \mu$ s of simulation. In section 3.C we show that this small crystallite, after annealing at $200 \mathrm{~K}$, has a welldefined melting temperature. Cooper et al. have discussed the effect of strong confinement on the kinetics of crystallization, and concluded that for very small (nanoscopic) volumes the crystallization is not limited by the nucleation barrier but by the availability of material to grow the critical nucleus into a stable crystallite. ${ }^{54,55}$ The formation of a stable crystallite from the small liquid plug of the $f=0.4$ pore indicates that this limit has not yet been reached for water domains containing about 650 water molecules at $180 \mathrm{~K}$.

B. Structure of Water in the Crystallized Nanopores. Figure 2 shows representative snapshots of the hydrophilic and hydrophobic pores with water filling fraction $f=0.6$, as they are heated up to their melting temperatures. The ice crystallized in the nanopore contains intercalated cubic and hexagonal layers (displayed in red and green, respectively, in Figure 2A). A structure with random stacks of hexagonal and cubic layer has been previously proposed in the literature for confined ice. ${ }^{1,12,14,41}$ Although the presence of profuse stacking faults in the ice crystallized in largely filled pores $(f>0.9)$ has been well established both in experiments ${ }^{11,12,14}$ and in simulations, ${ }^{43}$ it is not clear how the structure of ice depends upon pore filling. Recent neutron diffraction results for $7 \mathrm{~nm}$ diameter partially filled silica nanopores suggest that hexagonal ice is formed only for largely filled pores $(f>0.9)$, whereas for less filled pores $(f<$ $0.5)$, the characteristic triplet of hexagonal ice at low wave vectors $q$ was absent from the diffraction pattern. ${ }^{11}$ The absence of hexagonal peaks in the spectrum, however, should not be equated with an absence of hexagonal layers, as hexagonal growth and deformation stacking faults may account for as much as a third of the ice without the hexagonal ice signature peaks appearing in the diffraction pattern. ${ }^{56}$ We find an average ratio of cubic to hexagonal ice in the partially filled pores was around 2, same as for the filled pore in ref 43. This preference of cubic over hexagonal ice in confined water has been widely characterized. ${ }^{40,41,57}$ Hexagonal ice layers are still present in the simulations of the least filled pores, suggesting that they may be present in the experiments although they cannot be distinguished in the diffraction pattern.

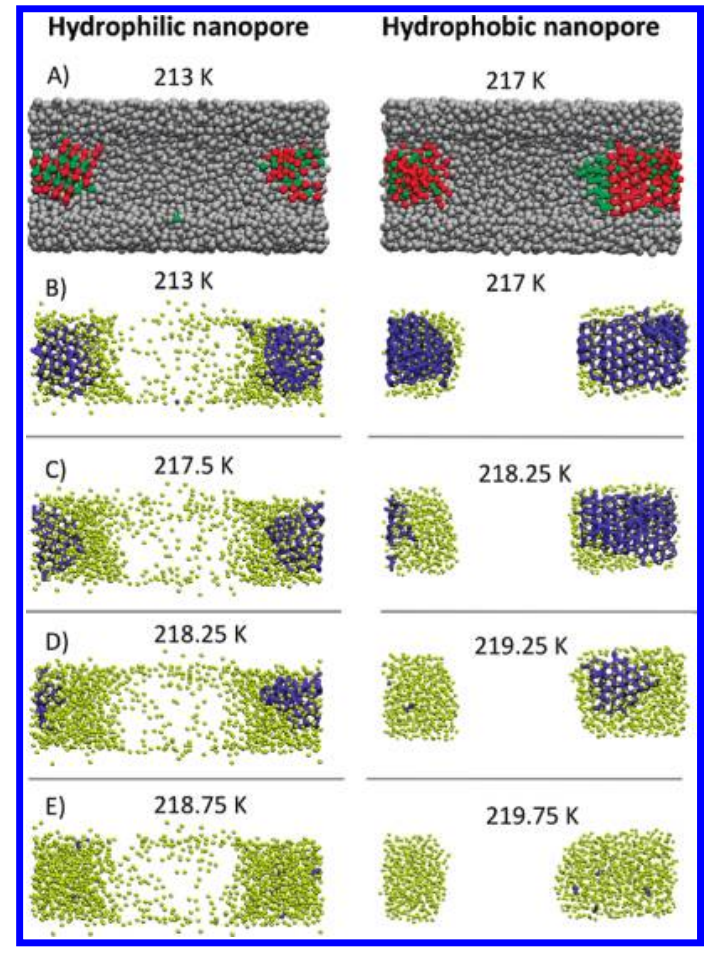

Figure 2. Melting of ice in 60\% filled hydrophilic and hydrophobic nanopores. Representative snapshots showing the progression of melting of confined ice on a heating ramp at a rate of $0.5 \mathrm{~K} \mathrm{~ns}^{-1}$ in pores with hydrophilic (left panel) and hydrophobic (right panel) walls at filling fraction $f=0.6$. Panel A: shows the pore wall (gray) and water molecules in the hexagonal (green) and cubic (red) ice. Panels B-E: water molecules in liquid water (yellow) and total ice (blue), where total ice includes cubic, hexagonal, and interfacial ice. It should be noted that the classification of the water molecules in the system in liquid or crystal is based on the correlation of bond-ordering and does not differentiate the water in the condensed liquid phase from the water adsorbed on the pore walls. The corresponding temperatures for each panel are indicated on top of each snapshot. Figure 3 shows the evolution of the fraction of ice with temperature in these two systems.

$\mathrm{NMR}$, calorimetry, and neutron and X-ray diffraction indicate that there is a noncrystallizable water fraction in nanopores. $5,11-14,16,58$ Our simulations indicate that this noncrystallizable water encompasses both small clusters of surface-adsorbed water (negligible for the hydrophobic pore wall) and the premelted liquid that surrounds the ice crystallites. Coexistence of ice, with its premelted liquid layer, and the surface-adsorbed phase in the hydrophilic nanopore can be appreciated in Figure 2. The surface adsorbed phase is distributed along the pore surface in the form of small water clusters, characterized in ref 47. These water clusters do not crystallize during the simulations; because of their small size and relatively low coverage of the surface, we do not expect the surface-adsorbed water to crystallize in experiments.

The confined ice in the pore is covered by a premelted liquid layer, for all pore fillings and pore-water interactions, down to the lowest temperature of this study, $180 \mathrm{~K}$. The presence of this liquid layer in coexistence with the ice plug at $T<T_{\mathrm{m}}$ is in agreement with NMR, neutron scattering, and X-ray reflectivity results and predictions from surface melting theory, ${ }^{11,12,19,59-64}$ and it has been observed in simulations of fully filled nanopores. 43,51 The fraction of water in the pore that does not crystallize at $180 \mathrm{~K}$ ranges from $80 \%$ for the least filled pore, to $40 \%$ for the fully filled 


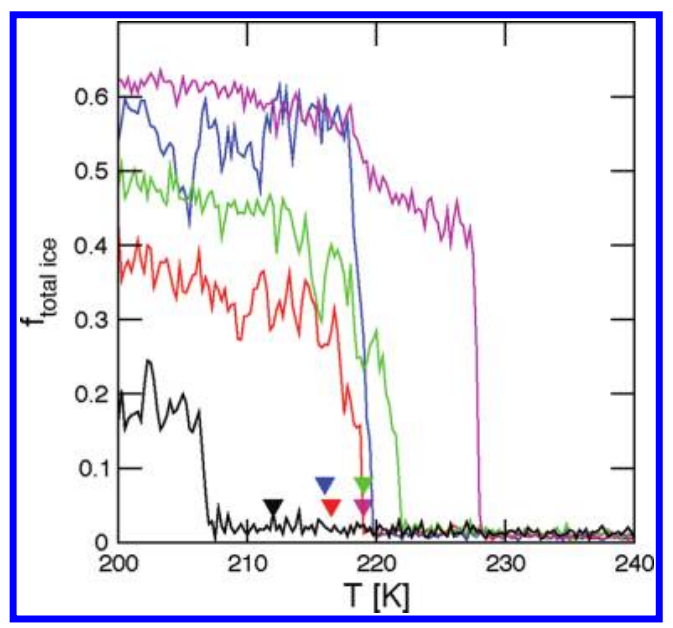

Figure 3. Fraction of water molecules in the ice phase upon heating the partially filled nanopores at a rate of $0.5 \mathrm{Kns}^{-1}$. Pore filling fractions $f$ are 1.0 (magenta), 0.9 (green), 0.6 hydrophobic (blue) and 0.6 hydrophilic (red), and 0.4 (black). It should be noted that the water in the pore is not in equilibrium along these warming up ramps; the fluctuations observed are a consequence of the heating rate employed. The triangles show the $T_{\mathrm{m}}$ for each system (same color code as the lines), determined from equilibrium simulations at a series of temperatures. The equilibrium melting temperatures are lower than the nonequilibrium melting temperatures, except for the least filled, $f=0.4$, pore for which significant annealing of the ice to a less defective structure occurs in the equilibrium simulations, resulting in a higher melting temperature.

one. The premelted liquid layer coexists in dynamical equilibrium with ice for extended periods of time, as can be seen in the microsecond simulations of the $40 \%$ filled system and the active exchange of molecules between ice and liquid (section 3.D below). The formation of such a surface-melted layer is inherent to the curvature and substrate effect of the confining matrix and arises because the liquid can accommodate better to the structure of the wall than the crystal. ${ }^{62,65,66}$ There is also a layer of liquid water between ice and vacuum at the two caps of the quasi-cylindrical crystallites, as expected from the known premelting of ice at a vapor interface. ${ }^{65,67,68}$

It has been argued that the presence of a nonfreezable liquid layer is due to the strong interactions with the pore walls. We find, however, a premelted liquid layer even in the highly hydrophobic pore. The effect of water-wall interaction in the premelted layer is addressed elsewhere, ${ }^{51}$ here we note that the fraction of ice in the hydrophobic nanopores is larger than in the hydrophilic nanopores (60\% ice for hydrophobic vs $35 \%$ ice for hydrophilic, both for $f=0.6$ ), due both to the lack of a significant surfaceadsorbed phase and a thinner premelted layer in the hydrophobic pores. In the next section we show that despite the differences in the surface-adsorbed and premelted layer for water confined in the hydrophobic and hydrophilic nanopores, the melting temperatures of ice in these two pores are the same.

C. Temperatures and Enthalpy of Melting of Ice in the Nanopores. A first estimation of the melting temperature of ice in the pores was obtained through the analysis of the crystallized systems resulting from the protocols described above, further annealed at 190 and $200 \mathrm{~K}$ for $50 \mathrm{~ns}$ and then heated from 200 to $240 \mathrm{~K}$ at a rate of $0.5 \mathrm{~K} \mathrm{~ns}^{-1}$. Figure 3 shows the fraction of water in the ice phase as the temperature increases. Melting along the temperature ramp was monitored through the fraction of molecules in the ice and liquid phases. The melting of ice is sharp for water in all the pores. Melting of the confined ice proceeds from

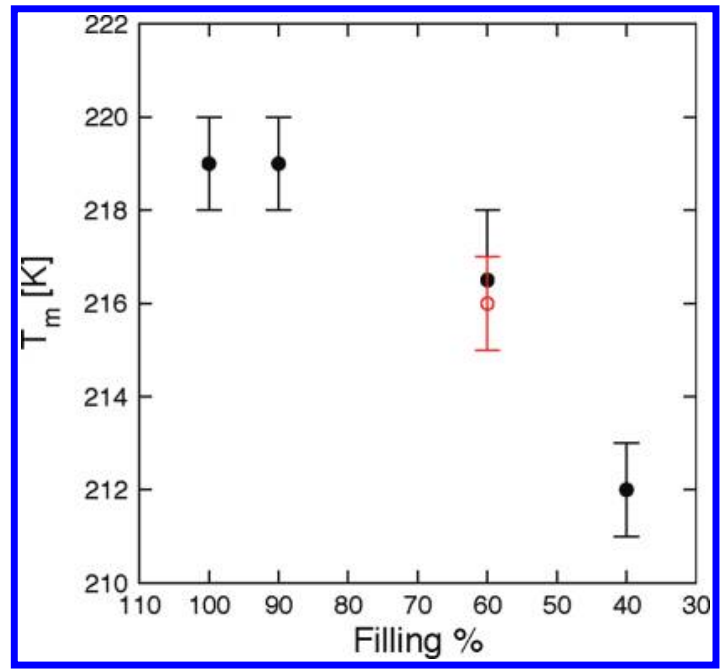

Figure 4. Melting temperature of ice as a function the percentage filling fraction. Black circles correspond to the hydrophilic pore and red to the hydrophobic pore wall, all of them of $3 \mathrm{~nm}$ diameter. The melting temperature depends strongly on the radius of the pore and weakly on the filling fraction and is insensitive to the hydrophobicity of the pore wall.

the outer part of the crystallite to the center, until the ice core reaches a size small enough for which it melts completely.

Accurate melting temperatures $\left(T_{\mathrm{m}}\right)$ were determined from a series of isothermal simulations, each $100 \mathrm{~ns}$ long, of the crystallized systems around the temperature for which ice melted during the heating ramp. The $T_{\mathrm{m}}$ are presented in Table 1 and graphed as a function of filling fraction in Figure 4. There is a slight drop in melting temperature, from 219 to $212 \mathrm{~K}$, as $f$ decreases from 1 to 0.4 . The decrease in $T_{\mathrm{m}}$ with filling fraction in the simulations is comparable to that reported in the experiments, 3-9 $\mathrm{K}^{11}$ Experimental studies of melting in nanopores using X-ray, ${ }^{64} \mathrm{NMR}^{11}{ }^{11} \mathrm{DSC},{ }^{5,11,69}$ and $\mathrm{ND}^{11}$ all agree that the melting temperature is not significantly affected by the level of filling of the pore. We attribute this small drift toward lower $T_{m}$ to an increase in the surface to volume ratio for the ice phase as the crystallite becomes smaller. We expect that longer pores with the same filling fraction would produce larger crystallites and thus present even less pronounced change in $T_{\mathrm{m}}$ with $f$. It should be noted, however, that this variation with $f$ is minimal when compared to the depression in $T_{\mathrm{m}}$ with respect to bulk ice, $T_{\mathrm{m}}{ }^{\text {bulk }}=274 \mathrm{~K}$ in the mW model. ${ }^{44}$ This implies that most of the free energy penalty to ice formation arises from the cylindrical interface of ice and not from the two circular areas that cap the ice plug.

We analyze the effect of water-surface interactions on the melting by comparing the hydrophobic and hydrophilic $f=0.6$ pores. We found that although the fraction of ice is considerably higher in the hydrophobic pore (Table 1 and Figure 3), the melting temperature of ice in both pores is the same (Table 1 and upper panel of Figure 4). These findings are in agreement with recent NMR cryoporometry and DSC results by Jelassi et al. that showed no difference in the melting temperatures of hydrophobic and hydrophilic partially filled and overfilled pores of $\sim 7 \mathrm{~nm}$ diameters $^{11}$ and DSC studies of fully filled nanopores by Findenegg et al. that show no difference in the $T_{\mathrm{m}}$ of ice in bare silica pores and the same pores with acid-functionalized surfaces. ${ }^{2}$ The structure of water in the two pores, however, is not the same: 
first, the premelted liquid layer is about $0.1 \mathrm{~nm}$ thinner and lower in density for the water in the hydrophobic pore and, second, there is no surface-adsorbed water in the hydrophobic pore. Therefore, agreement in the melting temperature for water confined in nanopores with different surface modifications must not be interpreted to indicate that the structure of confined water is the same. Our results concur with the recent diffraction study of Jelassi et al. that show that partially filled hydrophilic and hydrophobic nanopores that yield the same melting temperature, have nevertheless very different water structure. ${ }^{17}$

The enthalpy of melting $\Delta H_{\mathrm{m}}$ was computed from the difference of the enthalpy of water in the pore at the lowest temperature for which all water in the pore is liquid and the highest temperature for which there is ice in the pore. The enthalpy of melting was normalized in two ways: (i) by the total number of water molecules in the pore $\left(\Delta H_{\mathrm{m}}{ }^{\mathrm{w}}\right)$ and (ii) by the number of water molecules in the ice phase $\left(\Delta H_{\mathrm{m}}{ }^{\text {ice }}\right)$. The former allows for a direct comparison with the experiments, and the latter for an assessment of the actual enthalpy of melting per molecule undergoing the transition. The two values are presented in Table 1.

$\Delta H_{\mathrm{m}}{ }^{\mathrm{w}}$ increases with the filling fraction of the pore. This is a consequence of the increased fraction of water in the ice phase as $f$ increases. The enthalpy of melting normalized by the number of molecules actually undergoing the melting transition, $\Delta H_{\mathrm{m}}$ ice, on the other hand, is the same for all pores, irrespective of the filling fraction and whether they are hydrophobic or hydrophilic. The value of $\Delta H_{\mathrm{m}}{ }^{\text {ice }}, 1.00 \pm 0.05 \mathrm{kcal} \mathrm{mol}^{-1}$, is the one expected from the excess enthalpy of the liquid with respect to ice in bulk $\mathrm{mW}$ water at the temperature of melting of ice in the pores, $H^{\mathrm{ex}}=1.077$ and $0.977 \mathrm{kcal} / \mathrm{mol}$ at 220 and $210 \mathrm{~K}$, respectively. ${ }^{70}$ This value is lower than the bulk value of the $T_{\mathrm{m}}$ of bulk ice $(1.26 \mathrm{kcal} / \mathrm{mol}$ for $\mathrm{mW}$ ) because the structure of the liquid approaches that of lowdensity amorphous ice on cooling, which in turn is structurally very close to ice. ${ }^{6,45,70}$ The Gibbs-Thomson constant that emerges from this and other simulations studies of liquid-ice equilibria for $\mathrm{mW}$ water in hydrophilic cylindrical nanopores, ${ }^{43,51,71}$ $K_{\mathrm{GT}}=54 \mathrm{~K} \mathrm{~nm}$, is in excellent agreement with the value predicted from bulk water data at the bulk melting point. Nevertheless, our results indicate that the enthalpy of melting in the nanopore is lower than the bulk value at $273 \mathrm{~K}$. Our results lend support to the conjecture that, if the approximations used to derived the GibbsThomson constant are still valid in these narrow nanopores, then the agreement with the GT equation may reflect a compensation in the change in the enthalpy and surface tension at lower temperatures.

D. Exchange of Molecules between Premelted Liquid and Ice. Webber and co-workers investigated the state of water in $\sim 8.6 \mathrm{~nm}$ diameter overfilled SBA-15 silica pores through neutron diffraction and NMR relaxation at temperatures of 300 to $180 \mathrm{~K}$, the latter well below the $T_{\mathrm{m}}$ of ice in that pore, $260 \mathrm{~K} .{ }^{13,14}$ In addition to cubic and hexagonal ice they identified a signal that they assigned to a disordered component, termed "water/ice" or "plastic ice". Their results show that the transformation between ice and the disordered component is reversible with temperature, indicating that the partition of water between the ice and liquid layers is an equilibrium feature of the system. ${ }^{13}$ We interpret that the water/ice component in their study is the premelted liquid layer predicted by surface melting theory, ${ }^{19}$ which we observe in the simulations of this work and characterize in refs 43 and 51 .

Here we investigate the kinetics of the exchange of water molecules between the premelted liquid layer and the ice in the pore. We analyzed two $500 \mathrm{~ns}$ long isothermal simulations of the

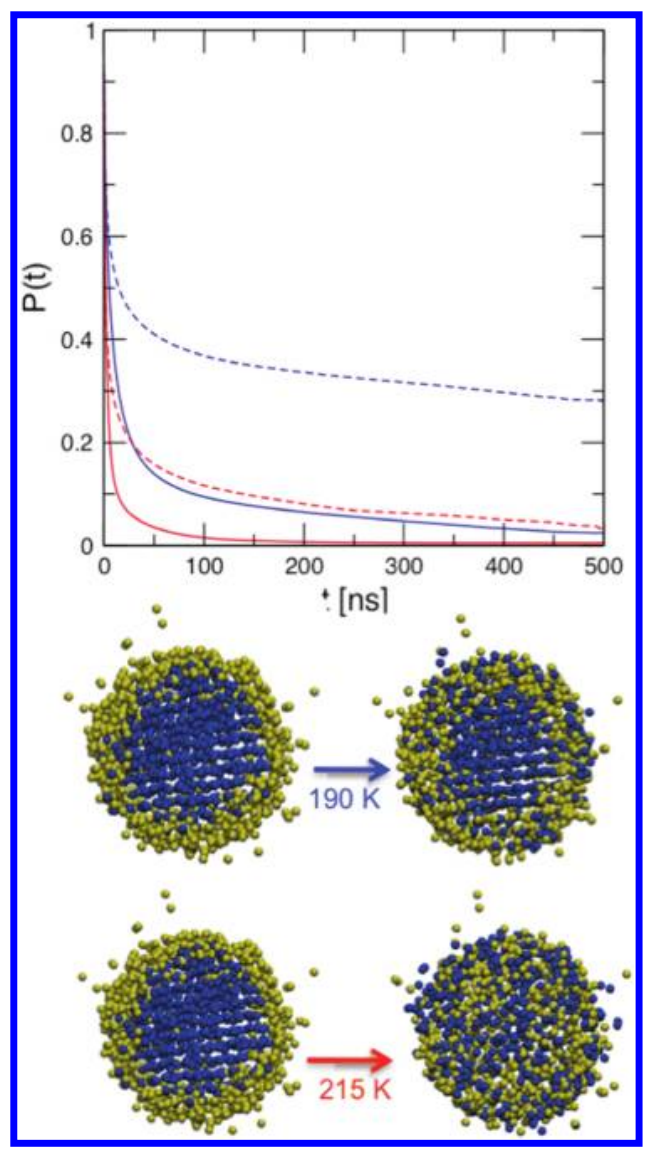

Figure 5. Exchange of water molecules between the premelted liquid and ice phases. The upper panel displays the probability that water molecules in the $60 \%$ filled hydrophilic nanopores remain in the liquid (full line) or crystal (dashed line) states at $190 \mathrm{~K}$ (blue) and $215 \mathrm{~K}$ (red). The lower panel shows views perpendicular to the pore axis for water in the pore at time $t=0$ (left snapshots) and after $500 \mathrm{~ns}$ (right snapshots) at these two temperatures. Molecules that originally were classified as part of the liquid are shown in yellow and molecules that at $t=0$ were classified as ice are shown in blue. Within 500 ns there is complete exchange of molecules between the two phases at $215 \mathrm{~K}$, but there is only partial exchange of the molecules at $190 \mathrm{~K}$.

$60 \%$ filled hydrophilic nanopore at 190 and $215 \mathrm{~K}$, both below its $T_{\mathrm{m}}=216 \mathrm{~K}$. The survival probability $P(t)$ of the molecules in the premelted liquid and ice are shown in the upper panel of Figure 5. The sharp initial decay of $P(t)$ during the first nanosecond is an artifact that arises from molecules oscillating in the liquid-crystal boundary and being alternatively classified as interfacial ice and as liquid without significant translation. Note that it is not mobility but change in identity as liquid or ice that is measured by $P(t)$ defined by eq 2 .

At $190 \mathrm{~K}$ the exchange was not fast enough to determine reliable time scales from the decay of $P(t)$ during the $500 \mathrm{~ns}$ long simulations. The molecules in the center of the crystal at $190 \mathrm{~K}$ do not exchange with the molecules that were initially in the premelted layer during the $500 \mathrm{~ns}$ of the simulations (lower panel of Figure 5). At $215 \mathrm{~K}$, on the other hand, the molecules in the interfacial region are mobile in the characteristic time scales of the exchange (lower panel of Figure 5). The survival probability of the molecules in the ice phase at $T=215 \mathrm{~K}$ was well represented by a stretched exponential decay (eq 3 ) with a very low exponent, $\beta=0.3$, which suggests a wide disitribution of 
relaxation times. The correlation time (eq 4) at $215 \mathrm{~K}$ was $130 \mathrm{~ns}$. The time scales of the mW model are known to be shorter than in experiments, particularly at low temperatures; ${ }^{44,46}$ thus the results of the simulations are a lower bound to the experimental exchange times.

Figure 5 shows that the exchange of molecules between liquid and ice at $215 \mathrm{~K}$ is accompanied by significant translation of the molecules in both the liquid and the ice. It is noteworthy that at $215 \mathrm{~K}\left(1 \mathrm{~K}\right.$ below $\left.T_{\mathrm{m}}\right)$ the ice core keeps its crystalline structure but practically all its molecules have exchanged with those in the liquid layer within the $500 \mathrm{~ns}$ of the simulations. We have not observed any significant water mobility in previous simulations of bulk ice with the mW model, ${ }^{44,49}$ suggesting that the diffusion of the molecules in the confined ice is facilitated by defects at the interface.

Our results indicate that there is a dynamic and reversible conversion between ice and the premelted layer in confined water at $T<T_{\mathrm{m}}$ and significant mobility not only in the liquid layer but also in the nanoconfined ice. The classification between liquid and crystal in this work is based on the analysis of the coherence of local ordering, and not the mobility of the molecules. The simulations indicate that the nonfreezable water located at the interface with the pore wall does not adopt a plastic ice structure but remains a disordered liquid. Our results suggest that the intermediate features between "liquid-like" and "solidlike" reported for nanoconfined water in ref 13 result from the reversible exchange between molecules in the premelted liquid layer and the ice crystal.

\section{CONCLUSIONS}

This works presents, to the best of our knowledge, the first study of freezing and melting of water in partially filled nanopores using molecular simulations. The use of an accurate and efficient coarse-grained model of water allowed us to perform simulations up to microseconds long, from which we characterized the dependence of the temperature and enthalpy of melting with filling fraction and hydrophobicity of the pore wall, the structure of the ice formed in nanoconfinement, the dynamic equilibrium between ice and a premelted liquid layer below the melting temperature, and the stochastic process of ice nucleation in partially filled nanopores. The simulations indicate that the crystallization of ice in partially filled pores occurs exclusively in the condensed liquid phase: the low-density surface-adsorbed water phase does not contribute to the formation of ice.

The ice-liquid equilibrium in nanopores differs from its bulk counterpart in at least two aspects. First, the melting temperature of ice in the nanopores is depressed with respect to the bulk $T_{\mathrm{m}}$. Second the confined ice in the pore coexists with a layer of liquidlike water that wets the pore surface down to at least $40 \mathrm{~K}$ below the equilibrium melting temperature. The simulations predict that the melting point of ice in the fully filled $3 \mathrm{~nm}$ diameter hydrophilic pore is $219 \pm 2 \mathrm{~K}$, in excellent agreement with the $215 \mathrm{~K}$ measured through differential scanning calorimetry for ice in $3 \mathrm{~nm}$ pore MCM-41 silica pores. ${ }^{3}$ The equilibrium melting temperatures for the confined ice in the nanopore were found to vary very slightly (within $9 \mathrm{~K}$ ) with pore filling, in good agreement with reports of experiments on MCM-41, SBA$15,{ }^{5,16}$ Vycor porous glass, ${ }^{69}$ and mesoporous silica. ${ }^{11}$

We find that the melting points of the confined ice are insensitive to the hydrophilicity/hydrophobicity of the pore wall, in agreement with recent experimental results for silica and functionalized silica nanopores. ${ }^{2,11}$ The water-pore surface interactions have, however, an important impact on the surface density $\Gamma$ of the adsorbed phase, and modulate the measured enthalpies of melting $\Delta H_{\mathrm{m}}$. The fraction of ice just below $T_{\mathrm{m}}$ in hydrophobic pores is larger than for hydrophilic pores, resulting in larger values of $\Delta H_{\mathrm{m}}$ per mole of water in the pore. The enthalpy of melting per mole of water in the pore is lower than for bulk water, in agreement with previous reports from experiments. ${ }^{2,3}$ The simulations indicate that this decrease in enthalpy of melting is not due to a loss of order in the crystalline phase but to the ordering of the liquid at low temperatures ${ }^{70}$ and to the significant fraction of liquid in the pore just below $T_{\mathrm{m}}$. When the enthalpy of melting is normalized by the number of water molecules in the crystal phase, the difference in enthalpy between liquid and ice in the nanopore is essentially identical to the excess enthalpy of bulk liquid water with respect to bulk ice at the melting temperature of ice in the pore.

There is a rounding of the ice-liquid transition in the $3 \mathrm{~nm}$ diameter pore, which results in a continuous increase in the fraction of liquid in the pore until it reaches the melting temperature. ${ }^{18,51}$ There are, however, distinct ice and liquid phases in the pore and the liquid-ice transition remains first-order-like. Some authors have attributed the NMR relaxation times measures for confined water below the melting temperature to a plastic crystalline state. ${ }^{13}$ Our results suggest that the intermediate dynamic properties of such a component, between liquid and crystal, arise from the continuous and reversible exchange of water molecules between the premelted liquid and the ice in the pores at a rate faster than discernible by the NMR and neutron diffraction measurements. ${ }^{13,14}$

\section{AUTHOR INFORMATION}

\section{Corresponding Author}

*E-mail Valeria.Molinero@utah.edu.

\section{ACKNOWLEDGMENT}

This work was supported by a collaborative research grant of the Agencia Nacional de Promoción Científica y Tecnológica de Argentina (V.M. and D.A.S.) and a Beckman Foundation Young Investigator Award (V.M.). E.G.S. and E.dlL. gratefully acknowledge doctoral fellowships by CONICET. We thank the Center of High Performance Computing of the University of Utah for allocation of computing time.

\section{REFERENCES}

(1) Brovchenko, I.; Oleinikova, A. Interfacial and Confined Water; Elservier: Amsterdam, 2008.

(2) Findenegg, G.; Jähnert, S.; Akcakayiran, D.; Schreiber, A. Chemphyschem 2008, 9, 2651.

(3) Jahnert, S.; Chavez, F. V.; Schaumann, G. E.; Schreiber, A.; Schonhoff, M.; Findenegg, G. H. Phys. Chem. Chem. Phys. 2008, 10, 6039.

(4) Schmidt, R.; Hansen, E.; Stöcker, M.; Akporiaye, D.; Ellestad, O. J. Am. Chem. Soc. 1995, 117, 4049.

(5) Schreiber, A.; Ketelsen, I.; Findenegg, G. H. Phys. Chem. Chem. Phys. 2001, 3, 1185.

(6) Kittaka, S.; Ishimaru, S.; Kuranishi, M.; Matsuda, T.; Yamaguchi, T. Phys. Chem. Chem. Phys. 2006, 8, 3223.

(7) Morishige, K.; Kawano, K. J. Chem. Phys. 1999, 110, 4867.

(8) Morishige, K.; Kawano, K. J Phys Chem B 1999, 103, 7906.

(9) Pearson, R. T.; Derbyshire, W. J. Colloid Interface Sci. 1974, 46, 232. 
(10) Hall, P. G.; Williams, R. T.; Slade, R. C. T. J. Chem. Soc., Faraday Trans. 1: Phys. Chem. Condens. Phases 1985, 81, 847.

(11) Jelassi, J.; Castricum, H. L.; Bellissent-Funel, M.-C.; Dore, J.; Webber, J. B. W.; Sridi-Dorbez, R. Phys. Chem. Chem. Phys. 2010, 12, 2838.

(12) Seyed-Yazdi, J.; Farman, H.; Dore, J. C.; Webber, J. B. W.; Findenegg, G. H.; Hansen, T. J. Phys.: Condens. Matter 2008, 20, 205108.

(13) Webber, J.; Dore, J.; Strange, J.; Anderson, R.; Tohidi, B. J. Phys.: Condens. Matter 2007, 19, 415117.

(14) Liu, E.; Dore, J. C.; Webber, J. B. W.; Khushalani, D.; Jähnert, S.; Findenegg, G. H.; Hansen, T. J. Phys.: Condens. Matter 2006, 18, 10009.

(15) Deschamps, J.; Audonnet, F.; Brodie-Linder, N.; Schoeffel, M.;

Alba-Simionesco, C. Phys. Chem. Chem. Phys. 2010, 12, 1440.

(16) Morishige, K.; Iwasaki, H. Langmuir 2003, 19, 2808.

(17) Jelassi, J.; Grosz, T.; Bako, I.; Bellissent-Funel, M.-C.; Dore, J. C.; Castricum, H. L.; Sridi-Dorbez, R. J. Chem. Phys. 2011, 134, 064509.

(18) Denoyel, R.; Pellenq, R. Langmuir 2002, 18, 2710.

(19) Petrov, O.; Furó, I. Phys. Rev. E: Stat., Nonlinear, Soft Matter Phys. 2006, 73, 011608.

(20) Koga, K.; Gao, G. T.; Tanaka, H.; Zeng, X. C. Nature 2001, $412,802$.

(21) Koga, K.; Gao, G. T.; Tanaka, H.; Zeng, X. C. Physica a-Stat. Mech. Its Applications 2002, 314, 462.

(22) Koga, K.; Parra, R. D.; Tanaka, H.; Zeng, X. C. J. Chem. Phys. 2000, 113, 5037.

(23) Koga, K.; Tanaka, H. J. Chem. Phys. 2005, 122, 104711.

(24) Koga, K.; Zeng, X.; Tanaka, H. Phys. Rev. Lett. 1997, 79, 5262.

(25) Bai, J.; Zeng, X. C.; Koga, K.; Tanaka, H. Mol. Simul. 2003, 29, 619 .

(26) Koga, K.; Tanaka, H.; Zeng, X. C. Nature 2000, 408, 564.

(27) Slovak, J.; Tanaka, H.; Koga, K.; Zeng, X. C. Physica a-Stat. Mech. Its Applications 2003, 319, 163.

(28) Zangi, R.; Mark, A. E. J. Chem. Phys. 2003, 119, 1694.

(29) Giovambattista, N.; Rossky, P. J.; Debenedetti, P. G. Phys. Rev. Lett. 2009, 102, 050603.

(30) Giovambattista, N.; Rossky, P. J.; Debenedetti, P. G. J. Phys. Chem. B 2009, 113, 13723.

(31) Giovambattista, N.; Rossky, P. J.; Debenedetti, P. G. Phys. Rev. E 2006, 73, 041604.

(32) Kumar, P.; Starr, F. W.; Buldyrev, S. V.; Stanley, H. E. Phys. Rev. E 2007, 75, 011202.

(33) Kumar, P.; Buldyrev, S. V.; Starr, F. W.; Giovambattista, N.; Stanley, H. E. Phys. Rev. E 2005, 72, 051503.

(34) Yamada, M.; Mossa, S.; Stanley, H.; Sciortino, F. Phys. Rev. Lett. 2002, 88, 195701.

(35) Kastelowitz, N.; Johnston, J. C.; Molinero, V. J. Chem. Phys. 2010, 132, 124511.

(36) Johnston, J. C.; Kastelowitz, N.; Molinero, V. J. Chem. Phys. 2010, 133, 154516.

(37) Bai, J.; Angell, C. A.; Zeng, X. C. Proc. Natl. Acad. Sci. U. S. A. 2010, 107, 5718.

(38) Han, S.; Choi, M. Y.; Kumar, P.; Stanley, H. E. Nat. Phys. 2010, 6,685 .

(39) Morishige, K.; Yasunaga, H.; Uematsu, H. J. Phys. Chem. C 2009, 113, 3056.

(40) Morishige, K.; Uematsu, H. J. Chem. Phys. 2005, 122, 044711.

(41) Baker, J. M.; Dore, J. C.; Behrens, P. J. Phys. Chem. B 1997, $101,6226$.

(42) Pereyra, R. G.; Carignano, M. A. J. Phys. Chem. C 2009, 113, 12699 .

(43) Moore, E. B.; de la Llave, E.; Welke, K.; Scherlis, D. A.; Molinero, V. Phys. Chem. Chem. Phys. 2010, 12, 4124.

(44) Molinero, V.; Moore, E. B. J. Phys. Chem. B 2009, 113, 4008.

(45) Moore, E. B.; Molinero, V. J. Chem. Phys. 2009, 130, 244505.

(46) Moore, E. B.; Molinero, V. J. Chem. Phys. 2010, 132, 244504.

(47) De La Llave, E.; Molinero, V.; Scherlis, D. A. J. Chem. Phys.

2010, 133, 034513.

(48) Stillinger, F. H.; Weber, T. A. Phys. Rev. B 1985, 31, 5262.
(49) Jacobson, L. C.; Hujo, W.; Molinero, V. J. Phys. Chem. B 2009, 113, 10298.

(50) Abascal, J. L. F.; Sanz, E.; Fernandez, R. G.; Vega, C. J. Chem. Phys. 2005, 122, 234511.

(51) Moore, E. B.; Allen, J. T.; Molinero, V. Liquid-ice coexistence below the melting temperature for water confined in hydrophilic and hydrophobic nanopores, to be submitted.

(52) Plimpton, S. J. J. Comput. Phys. 1995, 117, 1.

(53) Nelson, M. T.; Humphrey, W.; Gursoy, A.; Dalke, A.; Kale, L. V.; Skeel, R. D.; Schulten, K. Int. J. High Performance Comput. Appl. 1996, 10, 251.

(54) Cooper, S.; Nicholson, C.; Liu, J. J. Chem. Phys. 2008, 129, 124715 .

(55) Liu, J.; Nicholson, C. E.; Cooper, S. J. Langmuir 2007, 23, 7286.

(56) Moore, E. B.; Molinero, V. Is it cubic? Ice crystallization from deeply supercooled water. Phys. Chem. Chem. Phys. 2011, under review.

(57) Bellissent-Funel, M.-C.; Lal, J.; Bosio, L. J. Chem. Phys. 1993, 98, 4246.

(58) Petrov, O.; Furó, I. Microporous Mesoporous Mater. 2011, 138, 221.

(59) Petrov, O. V.; Furó, I. Prog. Nucl. Magn. Reson. Spectrosc. 2009, $54,97$.

(60) Wallacher, D.; Knorr, K. Phys. Rev. B 2001, 63, 104202.

(61) Engemann, S.; Reichert, H.; Dosch, H.; Bilgram, J.; Honkimaki, V.; Snigirev, A. Phys. Rev. Lett. 2004, 92, 205701.

(62) Parry, A. O.; Rascón, C.; Morgan, L. J. Chem. Phys. 2006, 124, 151101.

(63) Dash, J. G.; Rempel, A. W.; Wettlaufer, J. S. Rev. Mod. Phys. 2006, 78, 695 .

(64) Morishige, K.; Nobuoka, K. J. Chem. Phys. 1997, 107, 6965.

(65) Dash, J. G.; Rempel, A. W.; Wettlaufer, J. S. The physics of premelted ice and its geophysical consequences. Rev. Mod. Phys. 2006, 78,695 .

(66) Stewart, M. C.; Evans, R. Phys. Rev. E 2005, 71, 011602.

(67) Conde, M. M.; Vega, C.; Tribello, G. A.; Slater, B. J. Chem. Phys. 2009, 131, 034510.

(68) Bluhm, H.; Ogletree, D. F.; Fadley, C. S.; Hussain, Z.; Salmeron, M. J. Phys.: Condens. Matter 2002, 14, L227.

(69) Liu, X. X.; Wang, Q.; Huang, X. F.; Yang, S. H.; Li, C. X.; Niu, X. J.; Shi, Q. F.; Sun, G.; Lu, K. Q. J. Phys. Chem. B 2010, 114, 4145.

(70) Moore, E. B.; Molinero, V. Structural transformation in supercooled water controls the crystallization rate of ice. Nature 2011, arXiv:1107.1622v1 [cond-mast.soft], under review.

(71) De La Llave, E.; Gonzalez-Solveyra, E.; Scherlis, D. A.; Molinero, $\mathrm{V}$. Manuscript in preparation.

(72) CRC-Handbook of Handbook of Chemistry and Physics, 81st ed.; CRC Press: Boca Raton, FL, 2000-2001. 